\title{
Hattendorff Differential Equation for Multi-State Markov Insurance Models
}

\author{
Rajeev Rajaram ${ }^{*}+\mathbb{D}$ and Nathan Ritchey ${ }^{\dagger}$ \\ Department of Mathematical Sciences, Kent State University, Kent, OH 44242, USA; nritche2@kent.edu \\ * Correspondence: rrajaram@kent.edu \\ + These authors contributed equally to this work.
}

Citation: Rajaram, Rajeev, and Nathan Ritchey. 2021. Hattendorff Differential Equation for Multi-State Markov Insurance Models. Risks 9: 169. https://doi.org/ 10.3390/risks9090169

Academic Editor: Mogens Steffensen

Received: 9 July 2021

Accepted: 10 September 2021

Published: 16 September 2021

Publisher's Note: MDPI stays neutral with regard to jurisdictional claims in published maps and institutional affiliations.

Copyright: (c) 2021 by the authors. Licensee MDPI, Basel, Switzerland. This article is an open access article distributed under the terms and conditions of the Creative Commons Attribution (CC BY) license (https:/ / creativecommons.org/licenses/by/ $4.0 /)$.
Abstract: We derive a Hattendorff differential equation and a recursion governing the evolution of continuous and discrete time evolution respectively of the variance of the loss at time $t$ random variable given that the state at time $t$ is $j$, for a multistate Markov insurance model (denoted by ${ }^{2} \sigma_{t}^{(j)}$ ). We also show using matrix notation that both models can be easily adapted for use in MATLAB for numerical computations.

Keywords: policy value; Kolmogorov forward equations; multistate model; Thiele's differential equation; Hattendorff differential equation

\section{Introduction}

The original Hattendorff theorem in Hattendorff (1868) states that in a life insurance policy, the losses in different years are uncorrelated and have zero means, hence the variance of the total loss is the sum of variances of the losses in individual years. This result has had a revival in modern-day actuarial mathematics with a flavor of stochastic processes or a martingale approach as seen in Gerber $(1979,1986)$, Papatriandafylou and Waters (1984), Ramlau-Hansen (1988), Norberg (1992). Furthermore, the theorem has been mentioned in classic textbooks such as Bowers et al. (1986). An explicit integral formula for the variance of the loss at time $t$ random variable ${ }^{2} \sigma_{t}^{(j)}$, given that the state at time $t$ is $j$, was derived in Wolthuis (1987). Furthermore, Norberg (1995) and Asmussen and Steffensen (2020) derive differential equations for higher-order moments and variances in a more abstract setting. More recently in Bladt et al. (2020) a matrix representation was carried out to obtain representation formulas for all higher-order moments for the loss at time $t$ of the random variable $L_{t}$. In this paper, however, we explicitly derive models for the continuous and discrete time evolutions of the variance of $L_{t}$ under a much more general benefit and premium structure that is adaptable for numerical computation.

The main goals of this paper are as follows:

1. Explicitly derive a differential equation, which we name as the Hattendorff differential equation that governs the continuous time evolution of the variance of the loss at time $t$ random variable given that the state at time $t$ is $j$ (denoted by ${ }^{2} \sigma_{t}^{(j)}$ ), for a general setting of benefits and premiums in the setting of a multistate Markov insurance model.

2. Explicitly derive recursions for the discrete time evolution of the variance of the loss at time $t$ random variable ${ }^{2} \sigma_{t}^{(j)}$ (both annual and $h$-yearly cash flows), for a general setting of benefits and premiums in the context of a multistate Markov assumption.

3. Develop a matrix representation of the continuous time differential equation as well as the discrete time recursion (both annual and $h$-yearly cash flows) that allows for the use of MATLAB or similar software for ease of computation of ${ }^{2} \sigma_{t}^{(j)}$ as the solution of a backwards in time differential equation or a recursion.

4. Demonstrate the setup of the computations in the form of two examples in Section 6. 
Since the derivation of the models is a big task, we focus mainly on the derivation of the continuous and discrete time models in this paper for a general setup of benefits and premiums. The actual computation of ${ }^{2} \sigma_{t}^{(j)}$ for all time $t$ is a matter of solving a system of coupled differential equations. We tackle that issue in a separate paper in the future with numerical computations. However, we do demonstrate the usefulness of the two models derived by setting up the differential equation and the recursion respectively, for two situations. To our knowledge, this is the first time a general insurance model that is conducive for computation of the variance of loss ${ }^{2} \sigma_{t}^{(j)}$ has been derived.

In line with (Dickson et al. 2020, chp. 8), we consider a multistate model with $m+1$ states labeled as $0,1, \ldots, m$ with instantaneous transitions possible between pairs of states. We use $Y(t)$ to represent the state at time instant $t .\{Y(t)\}_{t>0}$ is a stochastic process for $t \geq 0$ (continuous or discrete time depending on when we choose to make observations), with $Y(t)=i$ to mean that the individual is in state $i$ at age $x+t$ (initial age is taken as $x$ ). We refer to Appendix A for notation and assumptions used in this paper.

We end this section by stating some $h$-yearly relationships between the $L_{t}^{(j)}$ and $L_{t+h^{\prime}}^{(j)}$ which can be easily derived by drawing a timeline for the interval $[t, t+h]$ and marking out the outgo and income:

$$
L_{t}^{(j)}=v^{h}\left(h B_{t+h}^{(j)}+S_{t+h}^{(j k)}+L_{t+h}^{(k)}\right)-h P_{t}^{(j)} \text { if } Y(t+h)=k \text { given } Y(t)=j .
$$

As a result, the $h$-yearly relationship between $V_{t}^{(j)}$ and $V_{t+h}^{j}$ can be derived by taking the expected value of Equation (1) as follows:

$$
V_{t}^{(j)}=v^{h} \sum_{k=0}^{m} h p_{x+t}^{j k}\left(h B_{t+h}^{(k)}+S_{t+h}^{(j k)}+V_{t+h}^{(k)}\right)-h P_{t}^{(j)}
$$

The paper is organized as follows. In Section 2, we explicitly derive discrete recursive relationships for ${ }^{2} \sigma_{t}^{(j)}$. In Section 3, we derive the differential equation describing the evolution of ${ }^{2} \sigma_{t}^{(j)}$ using the $h$-yearly recursion derived in Section 2. In Section 4 , we restate all the theorems with matrix notation. In Section 5, we briefly discuss possible terminal conditions that can be used to back-propagate the Hattendorff recursion and differential equation derived in Section 3. In Section 6 we apply the continuous time differential equation and the discrete time recursion to the disability insurance model to demonstrate how to set up the differential equation and the recursion respectively for computation using software. Finally, we conclude with some remarks in Section 7.

\section{Discrete Recursion for ${ }^{2} \sigma_{t}^{(j)}$}

In this section, we derive several discrete recursions for the variance ${ }^{2} \sigma_{t}^{(j)}$. First, we derive an $h$-yearly recursion for ${ }^{2} \sigma_{t}^{(j)}$.

Theorem 1. Assume that Notation A1, Assumption A1 and Notation A2 are true. Then, the following $h$-yearly recursion of ${ }^{2} \sigma_{t}^{(j)}$ holds.

$$
{ }^{2} \sigma_{t}^{(j)}=\sum_{k=0}^{m}{ }_{h} p_{x+t}^{j k} \cdot v^{2 h} \cdot{ }^{2} \sigma_{t+h}^{(k)}+\sum_{k \neq l}^{m}{ }_{h} p_{x+t}^{j k} p_{x+t}^{j l} v^{2 h}\left[\left(W_{t+h}^{(j k)}-W_{t+h}^{(j l)}\right]^{2}\right.
$$

Proof. Since,

$$
\left(L_{t}^{(j)}\right)^{2}=\left(v^{h}\left(h B_{t+h}^{(k)}+S_{t+h}^{(j k)}+L_{t+h}^{(k)}\right)-h P_{t}^{(j)}\right)^{2}, \text { with probability }{ }_{h} p_{x+t^{\prime}}^{j k}
$$


we know that

$$
\begin{aligned}
E\left(L_{t}^{(j)}\right)^{2} & =\sum_{k=0}^{m}{ }_{h} p_{x+t}^{j k} E\left(v^{h}\left(h B_{t+h}^{(k)}+S_{t+h}^{(j k)}+L_{t+h}^{(k)}\right)-h P_{t}^{(j)}\right)^{2} \\
& =\sum_{k=0}^{m}\left[h p_{x+t}^{j k} \cdot v^{2 h} \cdot{ }^{2} \sigma_{t+h}^{(k)}+{ }_{h} p_{x+t}^{j k}\left(v^{h} V_{t+h}^{(k)}+T^{j k}\right)^{2}\right],
\end{aligned}
$$

where $T^{j k}=v^{h}\left(h B^{(k)}+S_{t+h}^{(j k)}-h P_{t}^{(j)}\right)^{2}$ and ${ }^{2} \sigma_{t+h}^{(k)}=\operatorname{Var}\left(L_{t+h}^{(k)}\right)$.

Since the expected value is given by

$$
E\left(L_{t}^{(j)}\right)=\sum_{k=0}^{m} h p_{x+t}^{j k}\left(v^{h} V_{t+h}^{(k)}+T^{j k}\right)
$$

we have

$$
\left(E\left(L_{t}^{(j)}\right)\right)^{2}=\left(\sum_{k=0}^{m} h p_{x+t}^{j k}\left(v^{h} V_{t+h}^{(k)}+T^{j k}\right)\right)^{2} .
$$

Thus,

$$
\begin{aligned}
{ }^{2} \sigma_{t}^{(j)}=\operatorname{Var}\left(L_{t}^{(j)}\right) & =E\left(L_{t}^{(j)}\right)^{2}-\left(E\left(L_{t}^{(j)}\right)\right)^{2} \\
& =\sum_{k=0}^{m} h p_{x+t}^{j k} v^{2 h} \cdot{ }^{2} \sigma_{t+h}^{(k)} \\
& +\sum_{k=0}^{m} h p_{x+t}^{j k}\left(v^{h} V_{t+h}^{(k)}+T^{j k}\right)^{2}-\left(\sum_{k=0}^{m}{ }_{h} p_{x+t}^{j k}\left(v^{h} V_{t+h}^{(k)}+T^{j k}\right)\right)^{2} \\
& =\sum_{k=0}^{m} h p_{x+t}^{j k} v^{2 h} \cdot{ }^{2} \sigma_{t+h}^{(k)} \\
& +\sum_{k=0}^{m} h p_{x+t}^{j k}\left(U^{j k}\right)^{2}-\left(\sum_{k=0}^{m} h p_{x+t}^{j k} U^{j k}\right)^{2} \\
& =\sum_{k=0}^{m} h p_{x+t}^{j k} v^{2 h} \cdot{ }^{2} \sigma_{t+h}^{(k)}+\sum_{k \neq l}^{m}{ }_{h}^{j k} p_{x+t}^{j k}\left(U^{j k}-U^{j l}\right)^{2},
\end{aligned}
$$

where $U^{j k}=v^{h} V_{t+h}^{(k)}+T^{j k}$. Here, we have used the fact that $\sum_{k=0}^{m} p_{x+t}^{j k}=1$ in the last step above.

Noting that

$$
U^{j k}-U^{j l}=v^{h}\left[\left(V_{t+h}^{(k)}-V_{t+h}^{(l)}+h\left(B_{t+h}^{(k)}-B_{t+h}^{(l)}\right)+S_{t+h}^{(j k)}-S_{t+h}^{(j l)}\right],\right.
$$

we have

$$
\begin{aligned}
{ }^{2} \sigma_{t}^{(j)} & =\sum_{k=0}^{m} h p_{x+t}^{j k} \cdot v^{2 h} \cdot{ }^{2} \sigma_{t+h}^{(k)} \\
& +\sum_{k \neq l}^{m} h p_{x+t h}^{j k} p_{x+t}^{j l} v^{2 h}\left[\left(V_{t+h}^{(k)}-V_{t+h}^{(l)}+h\left(B_{t+h}^{(k)}-B_{t+h}^{(l)}\right)+S_{t+h}^{(j k)}-S_{t+h}^{(j l)}\right]^{2} .\right.
\end{aligned}
$$


So,

$$
{ }^{2} \sigma_{t}^{(j)}=\sum_{k=0}^{m}{ }_{h} p_{x+t}^{j k} \cdot v^{2 h} \cdot{ }^{2} \sigma_{t+h}^{(k)}+\sum_{k \neq l}^{m}{ }_{h} p_{x+t h}^{j k} p_{x+t}^{j l} v^{2 h}\left[\left(W_{t+h}^{(j k)}-W_{t+h}^{(j l)}\right]^{2}\right.
$$

Next, we obtain an annual recursion for ${ }^{2} \sigma_{t}^{(j)}$ as a corollary.

Corollary 1. Assume that Notation A1, Assumption A1 and Notation A2 are true. Then the following annual recursion for ${ }^{2} \sigma_{t}^{(j)}$ holds:

$$
\begin{aligned}
{ }^{2} \sigma_{t}^{(j)} & =\sum_{k=0}^{m} p_{x+t}^{j k} \cdot v^{2} \cdot{ }^{2} \sigma_{t+1}^{(k)}+\sum_{k \neq l}^{m} p_{x+t}^{j k} p_{x+t}^{j l} v^{2}\left[\left(W_{t+1}^{(j k)}-W_{t+1}^{(j l)}\right]^{2}\right. \\
& =p_{x+t}^{j j} v^{2} \cdot{ }^{2} \sigma_{t+1}^{(j)}+\sum_{\substack{k=0 \\
k \neq j}}^{m} p_{x+t}^{j k} v^{2} \cdot{ }^{2} \sigma_{t+1}^{(k)}+\sum_{k \neq l}^{m} p_{x+t}^{j k} p_{x+t}^{j l} v^{2}\left[\left(W_{t+1}^{(j k)}-W_{t+1}^{(j l)}\right]^{2} .\right.
\end{aligned}
$$

Proof. The proof follows by substituting $h=1$ in Theorem 1 .

Finally, we derive an explicit annual relationship for ${ }^{2} \sigma_{t}^{(j)}$ that mirrors the traditional Hattendorff recursion for the alive-dead model.

Theorem 2. Assume that Notation A1, Assumption A1 and Notation A2 are true. Then the following explicit annual relationship holds for ${ }^{2} \sigma_{t}^{(j)}$ :

$$
\begin{aligned}
{ }^{2} \sigma_{t+m}^{(j)}=v^{2 s} \prod_{i=0}^{s-1} p_{x+t+i}^{j j} \cdot{ }^{2} \sigma_{t+s}^{(j)}+\sum_{n=1}^{s} v^{2 n}\left(\prod_{i=0}^{n-2} p_{x+t+i}^{j j}\right) \sum_{\substack{k=0 \\
k \neq j}}^{i} p_{x+t+n-1}^{j k} \cdot{ }^{2} \sigma_{t+n}^{(k)} \\
+\sum_{n=1}^{s} v^{2 n}\left(\prod_{i=0}^{n-2} p_{x+t+i}^{j j}\right) \sum_{k \neq l}^{i} p_{x+t+n-1}^{j k} \cdot p_{x+t+n-1}^{j l}\left[\left(W_{t+n}^{(j k)}-W_{t+n}^{(j l)}\right]^{2}\right.
\end{aligned}
$$

\section{Proof.}

$$
\begin{gathered}
{ }^{2} \sigma_{t}^{(j)}=p_{x+t}^{j j} v^{2} \cdot{ }^{2} \sigma_{t+1}^{(j)}+\sum_{\substack{k=0 \\
k \neq j}}^{m} p_{x+t}^{j k} v^{2} \cdot 2 \sigma_{t+1}^{(k)} \\
+\sum_{k \neq l}^{m} p_{x+t}^{j k} p_{x+t}^{j l} v^{2}\left[\left(W_{t+1}^{(j k)}-W_{t+1}^{(j l)}\right]^{2}\right. \\
{ }^{2} \sigma_{t+1}^{(j)}=p_{x+t+1}^{j j} v^{2} \cdot{ }^{2} \sigma_{t+2}^{(j)}+\sum_{\substack{k=0 \\
k \neq j}}^{m} p_{x+t+1}^{j k} v^{2} \cdot{ }^{2} \sigma_{t+2}^{(k)} \\
+\sum_{k \neq l}^{m} p_{x+t+1}^{j k} p_{x+t+1}^{j l} v^{2}\left[\left(W_{t+2}^{(j k)}-W_{t+2}^{(j l)}\right]^{2}\right.
\end{gathered}
$$




$$
\begin{gathered}
{ }^{2} \sigma_{t+2}^{(j)}=p_{x+t+2}^{j j} v^{2} \cdot{ }^{2} \sigma_{t+3}^{(j)}+\sum_{\substack{k=0 \\
k \neq j}}^{m} p_{x+t+2}^{j k} v^{2} \cdot 2 \sigma_{t+3}^{(k)} \\
\quad+\sum_{k \neq l}^{m} p_{x+t+2}^{j k} p_{x+t+2}^{j l} v^{2}\left[\left(W_{t+3}^{(j k)}-W_{t+3}^{(j l)}\right]^{2}\right. \\
\vdots \\
{ }^{2} \sigma_{t+m}^{(j)}=p_{x+t+m}^{j j} v^{2} \cdot{ }^{2} \sigma_{t+m}^{(j)}+\sum_{\substack{k=0 \\
k \neq j}}^{m} p_{x+t+m}^{j k} v^{2} \cdot{ }^{2} \sigma_{t+m+1}^{(k)} \\
+\sum_{k \neq l}^{m} p_{x+t+m}^{j k} p_{x+t+m}^{j l} v^{2}\left[\left(W_{t+m+1}^{(j k)}-W_{t+m+1}^{(j l)}\right]^{2}\right.
\end{gathered}
$$

The theorem then follows by recursive substitution of the equations above.

\section{Hattendorff Differential Equation}

In this section, we derive a continuous time differential equation satisfied by ${ }^{2} \sigma_{t}^{(j)}$.

Theorem 3. Assume that Notation A1, Assumption A1 and Notation A2 are true. Then ${ }^{2} \sigma_{t}^{(j)}$ satisfies the following differential equation:

$$
\begin{array}{r}
\frac{d}{d t}{ }^{2} \sigma_{t}^{(j)}=\left(2 \delta+\sum_{k \neq j} \mu_{x+t}^{j k}\right) \cdot{ }^{2} \sigma_{t}^{(j)}-\sum_{k \neq j} \mu_{x+t}^{j k} \cdot{ }^{2} \sigma_{t}^{(k)} \\
-\sum_{l \neq j} \mu_{x+t}^{j l}\left(W_{t}^{(j j)}-W_{t}^{(j l)}\right)^{2} .
\end{array}
$$

Proof. Let us reconsider Theorem 1:

$$
{ }^{2} \sigma_{t}^{(j)}={ }_{h} p_{x+t}^{j j} v^{2 h} \cdot{ }^{2} \sigma_{t+h}^{(j)}+\sum_{\substack{k=0 \\ k \neq j}}^{m} h p_{x+t}^{j k} v^{2 h} \cdot{ }^{2} \sigma_{t+h}^{(k)}+\sum_{k \neq l} h p_{x+t}^{j k} \cdot{ }_{h} p_{x+t}^{j l} v^{2 h}\left[\left(W_{t+h}^{(j k)}-W_{t+h}^{(j l)}\right]^{2}\right.
$$

With a bit of algebra, we have

$$
\begin{aligned}
\frac{{ }^{2} \sigma_{t+h}^{(j)}-{ }^{2} \sigma_{t}^{(j)}}{h}={ }^{2} \sigma_{t+h}^{(j)}[ & \left.\frac{1-{ }_{h} p_{x+t}^{j j} v^{2 h}}{h}\right] \\
& -\sum_{\substack{k=0 \\
k \neq j}}^{m} \frac{h p_{x+t}^{j k}}{h} v^{2 h} \cdot{ }^{2} \sigma_{t+h}^{(k)}-\sum_{k \neq l} h p_{x+t}^{j k} \frac{h p_{x+t}^{j l}}{h} v^{2 h}\left[\left(W_{t+h}^{(j k)}-W_{t+h}^{(j l)}\right]^{2}\right.
\end{aligned}
$$

Of course, we will let $h$ approach zero and determine the limit of this expression. To organize our thoughts, note that 


$$
\lim _{h \rightarrow 0} \frac{{ }_{h} p_{x+t}^{j k}}{h}=\mu_{x+t}^{j k}, \quad \lim _{h \rightarrow 0} p_{x+t}^{j j}=1, \text { and } \lim _{h \rightarrow 0} p_{x+t}^{j k}=0 .
$$

We will first tackle the limit of the first term on the right. Please note that

$$
\frac{d}{d h}\left(v^{2 h}\right)=\frac{d}{d h}\left(e^{-2 \delta h}\right)=-2 \delta e^{-2 \delta h}=-2 \delta v^{2 h} .
$$

We will also need to recall Kolmogorov's Forward Equations. That is,

$$
\frac{d}{d t}\left({ }_{t} p_{x}^{j k}\right)=\sum_{k \neq j}\left[{ }_{t} p_{x}^{j k} \mu_{x+t}^{k j}-{ }_{t} p_{x}^{j j} \mu_{x+t}^{j k}\right]
$$

and hence

$$
\frac{d}{d h}\left({ }_{h} p_{x+t}^{j j}\right)=\sum_{k \neq j}\left[{ }_{h} p_{x+t}^{j k} \mu_{x+t+h}^{k j}-{ }_{h} p_{x+t}^{j j} \mu_{x+t+h}^{j k}\right] .
$$

We determine the following limit by applying L'Hospital's Rule.

$$
\begin{aligned}
\lim _{h \rightarrow 0} \frac{\left[1-{ }_{h} p_{x+t}^{j j} v^{2 h}\right]}{h} & =\lim _{h \rightarrow 0} \frac{\left[1-{ }_{h} p_{x+t}^{j j} e^{-2 \delta h}\right]}{h} \\
& =\lim _{h \rightarrow 0}-\left[{ }_{h} p_{x+t}^{j j}\left(-2 \delta v^{2 h}\right)+v^{2 h} \sum_{k \neq j}\left({ }_{h} p_{x+t}^{j k} \mu_{x+t+h}^{k j}-{ }_{h} p_{x+t}^{j j} \mu_{x+t+h}^{j k}\right)\right] \\
& =2 \delta+\sum_{j \neq k} \mu_{x+t}^{j k}
\end{aligned}
$$

As $h$ approaches 0, we have

$$
\begin{gathered}
\lim _{h \rightarrow 0} \sum_{\substack{k=0 \\
k \neq j}}^{m} \frac{h p_{x+t}^{j k}}{h} v^{2 h} \cdot{ }^{2} \sigma_{t+h}^{(k)}=\sum_{k \neq j}^{m} \mu_{x+t}^{j k} \cdot{ }^{2} \sigma_{t}^{(k)} \cdot \\
\frac{d}{d t}{ }^{2} \sigma_{t}^{(j)}=\left(2 \delta+\sum_{k \neq j} \mu_{x+t}^{j k}\right) \cdot{ }^{2} \sigma_{t}^{(j)}-\sum_{k \neq j} \mu_{x+t}^{j k} \cdot{ }^{2} \sigma_{t}^{(k)} \\
-\lim _{h \rightarrow 0} \sum_{k \neq j} \frac{h p_{x+t h}^{j k} p_{x+t}^{j l}{ }^{2 h}}{h}\left(\left(V_{t+h}^{(k)}-V_{t+h}^{(l)}\right)+h\left(B_{t+h}^{(k)}-B_{t+h}^{(l)}\right)+\left(S_{t+h}^{(j k)}-S_{t+h}^{(j l)}\right)\right)^{2} \\
=\left(2 \delta+\sum_{k \neq j} \mu_{x+t}^{j k}\right) \cdot{ }^{2} \sigma_{t}^{(j)}-\sum_{k \neq j} \mu_{x+t}^{j k} \cdot{ }^{2} \sigma_{t}^{(k)} \\
-\sum_{l \neq j} \mu_{x+t}^{j l}\left(\left(V_{t}^{(j)}-V_{t}^{(l)}\right)+\left(S_{t}^{(j j)}-S_{t}^{(j l)}\right)\right)^{2} \\
=\left(2 \delta+\sum_{k \neq j} \mu_{x+t}^{j k}\right) \cdot{ }^{2} \sigma_{t}^{(j)}-\sum_{k \neq j} \mu_{x+t}^{j k} \cdot{ }^{2} \sigma_{t}^{(k)} \\
-\sum_{l \neq j} \mu_{x+t}^{j l}\left(W_{t}^{(j j)}-W_{t}^{(j l)}\right)^{2} .
\end{gathered}
$$

This proves the theorem.

\section{Matrix Representation}

In this section, we rewrite some of the theorems in Sections 2 and 3 using the matrix notation introduced in Notation A3. This makes the equations look more elegant. 
First, we use matrix notation to restate Theorem 1.

Theorem 4. Assume that Notation A1, Assumption A1, Notation A2 and Notation A3 are true. Then the following $h$-yearly recursion for $\overrightarrow{{ }^{2} \sigma_{t}}$ holds:

$$
\overrightarrow{{ }^{2} \sigma_{t}}=v^{2 h} \cdot{ }_{h} \mathbb{P}_{x+t} \cdot{ }^{2} \overrightarrow{\sigma_{t+h}}+{ }^{2} \overrightarrow{\sigma_{t+h}^{d}}
$$

Proof. The proof follows by applying matrix notation in Notation A3 to Theorem 1.

We obtain the following corollary for annual recursion using $h=1$ in Theorem 4 .

Corollary 2. Assume that Notation A1, Assumption A1, Notation A2 and Notation A3 are true. The following annual recursion for $\overrightarrow{2} \sigma_{t}$ holds:

$$
\overrightarrow{{ }^{2} \sigma_{t}}=v^{2} \cdot \mathbb{P}_{x+t} \cdot{ }^{2} \overrightarrow{\sigma_{t+1}}+{ }^{2} \overrightarrow{\sigma_{t+1}^{d}}={ }^{2} \overrightarrow{\sigma_{t+1}^{d}}+{ }_{1}^{2} \mathbb{E}_{x+t}{ }^{2} \overrightarrow{\sigma_{t+1}} .
$$

Next, we obtain the matrix version of Theorem 2.

Theorem 5. Assume that Notation A1, Assumption A1, Notation $A 2$ and Notation A3 are true. Then the following explicit relationship for $\overrightarrow{{ }^{2} \sigma_{t}}$ holds:

$$
\overrightarrow{{ }^{2} \sigma_{t}}={ }_{s}^{2} \mathbb{E}_{x+t} 2 \overrightarrow{\sigma_{t+s}}+\sum_{l=0}^{s-1}{ }_{l}^{2} \mathbb{E}_{x+t}{ }^{2} \sigma_{t+l+1}^{\vec{d}} .
$$

Proof. Using the recursion from Corollary 2 we have

$$
\begin{gathered}
\overrightarrow{{ }^{2} \sigma_{t}}=v^{2} \cdot \mathbb{P}_{x+t} \cdot{ }^{2} \overrightarrow{\sigma_{t+1}}+{ }^{2} \overrightarrow{\sigma_{t+1}^{d}}={ }_{1}^{2} \mathbb{E}_{x+t}{ }^{2} \overrightarrow{\sigma_{t+1}}+{ }^{2} \sigma_{t+1}^{d} \\
{ }^{2} \overrightarrow{\sigma_{t+1}}=v^{2} \cdot \mathbb{P}_{x+t+1} \cdot{ }^{2} \overrightarrow{\sigma_{t+2}}+{ }^{2} \overrightarrow{\sigma_{t+2}^{d}}={ }_{1}^{2} \mathbb{E}_{x+t+1}{ }^{2} \overrightarrow{\sigma_{t+2}}+{ }^{2} \overrightarrow{\sigma_{t+2}^{d}} \\
{ }^{2} \overrightarrow{\sigma_{t+2}}=v^{2} \cdot \mathbb{P}_{x+t+2} \cdot{ }^{2} \overrightarrow{\sigma_{t+3}}+{ }^{2} \overrightarrow{\sigma_{t+3}^{d}}={ }_{1}^{2} \mathbb{E}_{x+t+2}{ }^{2} \overrightarrow{\sigma_{t+3}}+{ }^{2} \overrightarrow{\sigma_{t+3}^{d}}
\end{gathered}
$$

The theorem follows by recursively substituting these expressions into the first equation.

Finally, we rewrite Theorem 3 in matrix form.

Theorem 6. Assume that Notation A1, Assumption A1, Notation A2 and Notation A3 are true. Then, $\overrightarrow{2} \sigma_{t}$ satisfies the following differential equation.

$$
\frac{d}{d t}\left(\overrightarrow{{ }^{2} \sigma_{t}}\right)=\mathbb{M}_{x+t} \cdot \overrightarrow{{ }^{2} \sigma_{t}}-\overrightarrow{\mu_{s+t}^{d}}
$$

Proof. The theorem follows by applying Notation A3 to Theorem 3.

\section{Terminal Conditions}

In this section, we introduce terminal conditions and outline the steps involved to compute $\overrightarrow{2} \overrightarrow{\sigma_{t}}$ numerically. 


\section{1. $N$ Year Term or Endowment Insurance}

We consider end conditions for an $N$ year term (no survival benefit) or endowment insurance (survival benefit). In this case, all benefits at the end of the term are certain and hence the variance equals 0 . That is, $\overrightarrow{\sigma_{N}}=\overrightarrow{0}$.

\subsubsection{Annual Case}

For yearly cash flows, we have $s=N-t$ in Theorem 5

$$
\begin{aligned}
& \overrightarrow{2} \vec{\sigma}_{t}={ }_{N-t}^{2} \mathbb{E}_{x+t} \overrightarrow{{ }^{\sigma_{N}}}+\sum_{l=0}^{N-t-1}{ }_{l} \mathbb{E}_{x+t}{ }^{2} \sigma_{t+l+1}^{\vec{d}} \\
& =\sum_{l=0}^{N-t-1}{ }_{l}^{2} \mathbb{E}_{x+t}{ }^{2} \sigma_{t+l+1}^{\vec{d}} \\
& =2 \overrightarrow{\sigma_{t+1}^{d}}+\sum_{l=1}^{N-t-1}{ }_{l}^{2} \mathbb{E}_{x+t}{ }^{2} \sigma_{t+l+1}^{\vec{d}} \\
& ={ }^{2} \sigma_{t+1}^{d}+\sum_{p=0}^{N-t-2}{ }_{p+1} \mathbb{E}_{x+t}{ }^{2} \sigma_{t+p+2}^{\vec{d}} \quad \text { Let } p=l-1 \\
& ={ }^{2} \overrightarrow{\sigma_{t+1}^{d}}+\sum_{p=0}^{N-t-2}{ }_{1} \mathbb{E}_{x+t}{ }^{2} \mathbb{E}_{x+t+1}{ }^{2} \sigma_{t+p+2}^{\vec{d}} \\
& =2 \overrightarrow{\sigma_{t+1}^{d}}+{ }_{1}^{2} \mathbb{E}_{x+t} \sum_{p=0}^{N-t-2}{ }_{p}^{2} \mathbb{E}_{x+t+1}{ }^{2} \sigma_{t+p+2}^{\vec{d}} \\
& ={ }^{2}{\overrightarrow{\sigma_{t+1}^{d}}}_{1}+{ }_{1}^{2} \mathbb{E}_{x+t}{ }^{2} \overrightarrow{\sigma_{t+1}} \quad \text { follows from }\left(^{*}\right) .
\end{aligned}
$$

Thus,

$$
\overrightarrow{{ }^{2} \sigma_{t}}={ }^{2} \overrightarrow{\sigma_{t+1}^{d}}+{ }_{1}^{2} \mathbb{E}_{x+t}{ }^{2} \overrightarrow{\sigma_{t+1}}
$$

This equation can be used to go backwards in time recursively. Note: $\overrightarrow{t+1} V=\left[{ }_{t+1} V^{(0)}{ }_{t+1} V^{(1)}, \ldots, t+1 V^{(m)}\right]$ is needed to calculate ${ }^{2} \overrightarrow{\sigma_{t+1}^{d}}$ so this is coupled with the recursion for $\overrightarrow{t+1} V$.

We also note that from Equation $\left(^{*}\right)$ above we obtain the generalization of the scalar Hattendorff relationship that is widely celebrated to the multistate case.

Theorem 7. Assume that Notation A1, Assumption A1, Notation A2 and Notation A3 are true. Furthermore, assume an $\mathrm{N}$ - year term insurance for the multistate model for yearly cash flows. Then, the following relationship generalizes the discrete Hattendorff theorem for the alive-dead model:

$$
\overrightarrow{{ }^{2} \sigma_{t}}=\sum_{l=0}^{N-t-1}{ }_{l}^{2} \mathbb{E}_{x+t}{ }^{2} \sigma_{t+l+1}^{\vec{d}} .
$$

\subsubsection{Continuous Case}

Recall that the Hattendorff differential equation derived earlier in Theorem 3 is

$$
\frac{d}{d t} \overrightarrow{2} \sigma_{t}=\mathbb{M}_{x+t} \overrightarrow{{ }^{2} \sigma_{t}}-\overrightarrow{\mu_{x+t}^{d}} .
$$

For an $N$ year term or endowment insurance, $\overrightarrow{\sigma_{N}}=\overrightarrow{0}$, so we can use this fact to solve the differential equation backwards in time with this as end conditions. 
In general, we will need to use Matlab or similar software to compute the solution.

Let $\Phi\left(t, t_{0}\right)$ be the fundamental solution, which can be computed separately and stored, as follows.

$$
\frac{d}{d t}\left(\Phi\left(t, t_{0}\right)\right)=\mathbb{M}_{x+t} \cdot \Phi\left(t, t_{0}\right) ; \quad \text { with } \quad \Phi\left(t_{0}, t_{0}\right)=I_{m+1}
$$

Please note that $\Phi\left(t, t_{0}\right)$ is invertible for all $t \geq 0$.

Additionally,

$$
\overrightarrow{{ }^{2} \sigma_{t}}=\Phi\left(t, t_{0}\right){ }^{2} \sigma_{t_{0}}-\int_{t_{0}}^{t} \Phi\left(t_{0}+t-s, t_{0}\right) \overrightarrow{\mu_{x+s}^{d}} d s .
$$

In other words,

$$
\overrightarrow{{ }^{2} \sigma_{0}}=\Phi^{-1}\left(t, t_{0}\right) \overrightarrow{{ }^{2} \sigma_{t}}+\Phi^{-1}\left(t, t_{0}\right) \int_{t_{0}}^{t} \Phi\left(t_{0}+t-s, t_{0}\right) \overrightarrow{\mu_{x+s}^{d}} d s .
$$

Again, for an $N$ year term or endowment insurance, ${ }^{2} \overrightarrow{\sigma_{N}}=\overrightarrow{0}$, so with $t=N$ above, we have the following:

$$
\overrightarrow{{ }^{2} \sigma_{0}}=\Phi^{-1}\left(t, t_{0}\right) \int_{t_{0}}^{t} \Phi\left(t_{0}+t-s, t_{0}\right) \overrightarrow{\mu_{x+s}^{d}} d s .
$$

The above equation generalizes the scalar Hattendorff equation to the multistate continuous case, so we state it as a theorem below:

Theorem 8. Assume that Notation A1, Assumption A1, Notation A2 and Notation A3 are true. Furthermore, assume an $\mathrm{N}$-year term insurance for the multistate model for continuous cash flows. Then, the following relationship generalizes the discrete Hattendorff theorem for the alive-dead model:

$$
\overrightarrow{{ }^{2} t_{0}}=\Phi^{-1}\left(t, t_{0}\right) \int_{t_{0}}^{t} \Phi\left(t_{0}+t-s, t_{0}\right) \overrightarrow{\mu_{x+s}^{d}} d s .
$$

We can use any of the quadrature approximation formulas to compute $\overrightarrow{\sigma_{t_{0}}}$ as a function of $t_{0}$ (or $\overrightarrow{{ }^{2} \sigma_{t}}$ as a function of $t$ ) in Equation 6. Alternatively, the solution of the differential equation in Equation (5) can be directly computed backwards in time using MATLAB or similar software with the terminal conditions ${ }^{2} \overrightarrow{\sigma_{N}}=\overrightarrow{0}$.

Please note that

$$
\Phi^{-1}\left(t, t_{0}\right)=e^{-2 \delta\left(t-t_{0}\right)} I_{m+1} J\left(t, t_{0}\right)
$$

where $J$ is a matrix.

\subsubsection{Alive-Dead Model Continuous Case}

The discrete case for Hattendorff recursion is well known for the alive-dead model. We choose $m=0$ in Theorem 3 to derive the scalar counterpart of Theorem 3 as follows:

$$
\frac{d}{d t}\left({ }^{2} \sigma_{t}\right)=\left(2 \delta+\mu_{x+t}\right)^{2} \sigma_{t}-\mu_{x+t}\left(S_{t}-{ }_{t} V\right)^{2}
$$

Let

$$
I(t)=e^{-\int_{t_{0}}^{t}\left(2 \delta+\mu_{x+s}\right) d s}
$$

Then,

$$
\frac{d}{d t}\left({ }^{2} \sigma_{t}\right) e^{-\int_{t_{0}}^{t}\left(2 \delta+\mu_{x+s}\right) d s}=-e^{-\int_{t_{0}}^{t}\left(2 \delta+\mu_{x+s}\right) d s} \mu_{x+t}\left(S_{t}-{ }_{t} V\right)^{2}
$$


whose solution is

$$
\left.{ }^{2} \sigma_{t_{0}}={ }^{2} \sigma_{t} v^{2\left(t-t_{0}\right)}{ }_{t-t_{0}} p_{x+t_{0}}+\int_{t_{0}}^{t} v^{2\left(u-t_{0}\right)} u-t_{0} p_{x+t_{0}}\left(S_{u}-{ }_{u} V\right)^{2}\right) d u .
$$

For an $N$ year term or endowment insurance: ${ }^{2} \sigma_{N}=0$.

Thus,

$$
\left.{ }^{2} \sigma_{t_{0}}=\int_{t_{0}}^{t} v^{2\left(u-t_{0}\right)} u-t_{0} p_{x+t_{0}}\left(S_{u}-{ }_{u} V\right)^{2}\right) d u,
$$

which can again be computed using any of the quadrature approximation formulas. Alternatively, the differential equation (Equation (7)) can be directly solved backwards in time using the terminal condition ${ }^{2} \sigma_{N}=0$.

\subsection{Whole Life Insurance}

We now specialize the previous results for the case of whole life insurance both for discrete and continuous time.

\subsubsection{Annual Case}

For yearly cash flows, we have the following:

$$
\overrightarrow{{ }^{2} \sigma_{t}}={ }_{N-t}^{2} \mathbb{E}_{x+t} \overrightarrow{2}_{N}+\sum_{l=0}^{N-t-1}{ }_{l}^{2} \mathbb{E}_{x+t} 2 \sigma_{t+l+1}^{\vec{d}} .
$$

For whole life insurance, it is reasonable to assume that as $N$ approaches infinity,

$$
\left\|\mathbb{E}_{N+t}^{2} \overrightarrow{\sigma_{N}}\right\| \rightarrow 0
$$

Then we have the following generalization of Hattendorff equation for the multistate insurance model:

$$
\overrightarrow{{ }^{2} \sigma_{t}}=\sum_{l=0}^{\infty}{ }_{l}^{2} \mathbb{E}_{x+t}{ }^{2} \sigma_{t+l+1}^{\vec{d}} .
$$

To compute $\overrightarrow{2} \sigma_{t}$, we could choose $N$ large enough and compute the backward recursion given by Equation (8) backwards in time with the end condition ${ }^{2} \sigma_{N}=\overrightarrow{0}$.

\subsubsection{Continuous Case}

We recall the variation of parameters solution formula for the differential equation given by Equation (5):

$$
\overrightarrow{{ }^{2} \sigma_{0}}=\Phi^{-1}\left(t, t_{0}\right) \overrightarrow{{ }^{2} \sigma_{t}}+\Phi^{-1}\left(t, t_{0}\right) \int_{t_{0}}^{t} \Phi\left(t+t_{0}-s, t_{0}\right) \overrightarrow{\mu_{x+s}^{d}} d s .
$$

We can reasonably assume that $\left\|\Phi^{-1}\left(t, t_{0}\right) \overrightarrow{{ }^{2} \sigma_{t}}\right\| \rightarrow 0$ as $t \rightarrow \infty$, especially since $\Phi^{-1}\left(t, t_{0}\right)=e^{-2 \delta\left(t-t_{0}\right)} I_{m+1} J\left(t, t_{0}\right)$. Then, we have the following:

$$
\overrightarrow{2 \sigma_{t_{0}}}=\Phi^{-1}\left(t, t_{0}\right) \int_{t_{0}}^{\infty} \Phi\left(t+t_{0}-s, t_{0}\right) \overrightarrow{\mu_{x+s}^{d}} d s .
$$

We can use quadrature formulas to compute the above equation. Alternatively, we could also choose $T$ large enough and just simulate the solution of the ordinary differential equation given by Equation (5) backwards in time with $\overrightarrow{{ }^{2} \sigma_{T}}=\overrightarrow{0}$ as end conditions. This, in turn will be a good approximation. 
The above work illustrates that we have extended the Hattendorff differential equation that describes a life that makes transitions to multiple states. During that process, a Hattendorff recursion for the annual and $h$-yearly evolution of ${ }^{2} \sigma_{t}^{(j)}$ for annual cash and $h$-yearly cash flows respectively, along with terminal conditions has been developed.

\section{Examples}

In this section, we demonstrate how we can set up the recursion or differential equation as the case might be, with two examples. We have deliberately chosen not to carry out computations because software such as MATLAB or something similar can be used to compute solutions of differential equations and recursions very easily given the terminal conditions. The goal is to use the given basis in the examples to set up the differential equation or recursion for the model illustrated in Figure 1. We have deliberately avoided explicit numerical calculations as it is our intention to demonstrate numerical experiments in a future paper.

In both examples in this section, we use the disability income insurance model shown in Figure 1, where state 0 is the "alive" state, state 1 is "disabled" where the disability is temporary that can be moved out of, and state 2 is "Dead". State 0 and 1 can be visited multiple times (indicated by the double arrow); however, state 2 is an absorbing state, i.e., once in state 2 , the process terminates. We also use the standard actuarial mathematics halo notation where $\mu_{x}^{i j}$ stands for the force of transition for a life aged $x$ from state $i$ to state $j, \bar{a}_{x+t}^{i j}$ is the expected present value of benefit for a life aged $x$ for being in state $j$ at time $t$ that was initially in state $i$ where the benefit is paid immediately, and $\bar{A}_{x+t}^{i j}$ is the expected present value of a transition benefit for a life aged $x$ that was originally in state $i$ at time 0 and has transitioned to state $j$ at time $t$. The values chosen for the insurance basis in both problems are typical values in actuarial mathematics. Our main goal in this section is to demonstrate the usefulness of the two models derived by showing that they can be rewritten in the form of coupled differential or difference equations that can be used for computations easily.

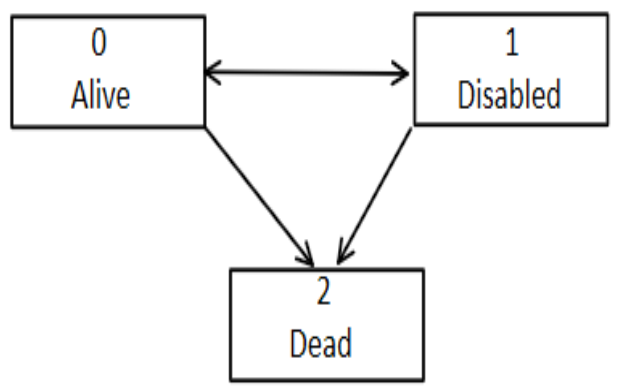

Figure 1. Temporary disability income insurance model.

\subsection{Example 1: Continuous Time Disability Income Insurance Model}

Consider a disability income insurance model with the following transition rates:

$$
\mu_{60+t}^{01}=0.05, \quad \mu_{60+t}^{02}=0.25 t, \quad \mu_{60+t}^{10}=0.25, \quad \mu_{60+t}^{12}=0.04 t
$$

The valuation force of interest is $5 \%$. You are also given:

$$
\bar{a}_{60}^{00}=5.1716, \quad \bar{a}_{60}^{01}=0.8430, \quad \bar{A}_{60}^{02}=0.6980, \quad \bar{a}_{60}^{11}=4.8201, \quad \bar{A}_{60}^{12}=0.7350,
$$




$$
\bar{a}_{70}^{00}=2.4769, \quad \bar{a}_{70}^{01}=0.2012, \quad \bar{A}_{70}^{02}=0.8659, \quad \bar{a}_{70}^{10}=0.1051, \quad \bar{a}_{70}^{11}=1.8528, \quad \bar{A}_{70}^{12}=0.9017,
$$

and

$$
{ }_{10} p_{60}^{00}=0.18314,{ }_{10} p_{60}^{01}=0.06181 .
$$

A 10-year health insurance product has the following features:

- $\quad$ The product is issued to lives aged 60 in the healthy state.

- $\quad$ The product pays a death benefit of 5000 at the moment of death.

- The product pays a continuous disability benefit at a rate of 750 per year while the insured is temporarily disabled.

- $\quad$ Net premiums are payable continuously while the insured is healthy.

- The product pays an endowment of 1000 if the person lives beyond 10 years.

The net premium can be computed using the Equivalence Premium Principle that is assumed to be at a rate of $P=695.64$.

By Thiele's differential equation, we have the following:

$$
\begin{aligned}
\frac{d}{d t}\left({ }_{t} V^{(0)}\right) & =\delta_{t} V^{(0)}+P-\left\{\mu_{60+t}^{01}\left(S_{t}^{(01)}+{ }_{t} V^{(1)}-{ }_{t} V^{(0)}\right)+\mu_{60+t}^{02}\left(S_{t}^{(02)}+{ }_{t} V^{(2)}-{ }_{t} V^{(0)}\right)\right\} \\
& =(0.05) \cdot{ }_{t} V^{(0)}+695.64-\left\{0.05\left(0+{ }_{t} V^{(1)}-{ }_{t} V^{(0)}\right)+0.025 t\left(5000+{ }_{t} V^{(2)}-{ }_{t} V^{(0)}\right)\right\} \\
& =695.64+{ }_{t} V^{(0)}(0.05+0.05+0.025 t)-0.05{ }_{t} V^{(1)}-(0.025 t)(5000) \\
& =695.64+{ }_{t} V^{(0)}(0.1+0.025 t)-0.05 \cdot{ }_{t} V^{(1)}-125 t .
\end{aligned}
$$

We also have the following for ${ }_{t} V^{(1)}$ :

Please note that $t_{t} V^{(2)}=0$ for all $t \geq 0$.

$$
\begin{aligned}
\frac{d}{d t}\left({ }_{t} V^{(1)}\right) & =\delta_{t} V^{(1)}-B_{t}^{(1)}-\left\{\mu \mu_{60+t}^{10}\left(S_{t}^{(10)}+{ }_{t} V^{(0)}-{ }_{t} V^{(1)}\right)+\mu \mu_{60+t}\left(S_{t}{ }^{(12)}+{ }_{t} V^{(2)}-{ }_{t} V^{(1)}\right)\right\} \\
& =(0.05) \cdot{ }_{t} V^{(1)}+750-\left\{0.025\left(0+{ }_{t} V^{(0)}-{ }_{t} V^{(1)}\right)+0.04 t\left(5000-{ }_{t} V^{(1)}\right)\right\} \\
& =(0.05+0.025+0.04 t) \cdot{ }_{t} V^{(1)}-0.025{ }_{t} V^{(0)}-750-200 t \\
& =(0.075+0.04 t) \cdot{ }_{t} V^{(1)}-0.025{ }_{t} V^{(0)}-(750-200 t) .
\end{aligned}
$$

Thus, Thiele's differential equation can be written as Since ${ }_{t} V^{(2)}=0$ for all $t \geq 0$ we have no need for a differential equation.

$$
\begin{gathered}
\frac{d}{d t}\left[\begin{array}{l}
{ }_{t} V^{(0)} \\
{ }_{t} V^{(1)}
\end{array}\right]=\left[\begin{array}{cc}
0.1+0.025 t & -0.05 \\
-0.025 & 0.075+0.04 t
\end{array}\right]\left[\begin{array}{l}
{ }_{t} V^{(0)} \\
{ }_{t} V^{(1)}
\end{array}\right]+\left[\begin{array}{c}
-125 t \\
695.64-(750+200 t)
\end{array}\right], \\
\text { Terminal Conditions: }\left\{\begin{array}{l}
{ }_{10} V^{(0)}=1000 \\
{ }_{10} V^{(1)}=0
\end{array}\right. \text { (Endowment) }
\end{gathered}
$$

Now let us consider Hattendorff's differential equation.

Recall that $\delta=0.05$ so $2 \delta=0.1$.

Now,

$$
\begin{aligned}
\frac{d}{d t}\left[\begin{array}{c}
{ }^{2} \sigma_{t}^{(0)} \\
{ }^{2} \sigma_{t}^{(1)} \\
{ }^{2} \sigma_{t}^{(2)}
\end{array}\right]=\left[\begin{array}{ccc}
\left(2 \delta+\mu_{60+t}^{01}+\mu_{60+t}^{02}\right) & -\mu_{60+t}^{01} & -\mu_{60+t}^{02} \\
-\mu_{60+t}^{10} & \left(2 \delta+\mu_{60+t}^{10}+\mu_{60+t}^{12}\right) & -\mu_{60+t}^{12} \\
0 & 0 & 2 \delta
\end{array}\right]\left[\begin{array}{c}
2 \sigma_{t}^{(0)} \\
2 \sigma_{t}^{(1)} \\
2 \sigma_{t}^{(2)}
\end{array}\right] \\
-\left[\begin{array}{c}
\sum_{l \neq 0} \mu_{60+t}^{0 l}\left(V_{t}^{(0)}-\left(V_{t}^{(l)}-S_{t}^{(0 l)}\right)\right)^{2} \\
\sum_{l \neq 1} \mu_{60+t}^{1 l}\left(V_{t}^{(1)}-\left(V_{t}^{(l)}-S_{t}^{(1 l)}\right)\right)^{2} \\
\sum_{l \neq 2} \mu_{60+t}^{2 l}\left(V_{t}^{(2)}-\left(V_{t}^{(l)}-S_{t}^{(2 l)}\right)\right)^{2}
\end{array}\right] .
\end{aligned}
$$


Additionally,

$$
\begin{gathered}
S_{t}^{(01)}=0, S_{t}^{(02)}=5000, S_{t}^{(10)}=0, S_{t}^{(12)}=5000, \\
W_{t}^{j j}=V_{t}^{(j)}-S_{t}^{(j j)}=V_{t}^{(j)}, W_{t}^{00}=V_{t}^{(0)},
\end{gathered}
$$

and

$$
W_{t}^{(j l)}=V_{t}^{(l)}-S_{t}^{(j l)} .
$$

Therefore,

$$
\begin{aligned}
\frac{d}{d t}\left[\begin{array}{l}
2 \sigma_{t}^{(0)} \\
2 \sigma_{t}^{(1)} \\
2 \sigma_{t}^{(2)}
\end{array}\right]= & {\left[\begin{array}{ccc}
0.1+0.05+0.025 t & -0.05 & -0.025 t \\
-0.025 & 0.1+0.025+0.04 t & -0.04 t \\
0 & 0 & 0.1
\end{array}\right]\left[\begin{array}{c}
2 \sigma_{t}^{(0)} \\
2 \sigma_{t}^{(1)} \\
2 \sigma_{t}^{(2)}
\end{array}\right] } \\
- & {\left[\begin{array}{l}
0.05\left(V_{t}^{(0)}-V_{t}^{(1)}\right)^{2}+0.025 t\left(5000+V_{t}^{(0)}-V_{t}^{(2)}\right)^{2} \\
0.025\left(V_{t}^{(1)}-V_{t}^{(0)}\right)^{2}+0.04 t\left(5000+V_{t}^{(1)}-V_{t}^{(2)}\right)^{2} \\
0
\end{array}\right] }
\end{aligned}
$$

Terminal Conditions are as follows:

$$
{ }^{2} \sigma_{10}^{(0)}={ }^{2} \sigma_{10}^{(1)}={ }^{2} \sigma_{10}^{(2)}=0 .
$$

We end the example by stating the following:

1. ${ }_{t} V^{(j)}$ can be solved by solving Thiele's differential equation backwards in time with terminal conditions. The solution can be stored in memory.

2. ${ }^{2} \sigma_{t}^{(j)}$ can be solved by solving Hattendorff's differential equation backwards in time with terminal conditions. This step will require the solution of Thiele's differential equation.

3. Both the previous steps can be easily accomplished using MATLAB or similar software.

\subsection{Example 2: Discrete Time Disability Income Insurance Model}

Here are the assumptions for this case.

1. Assume same $\mu^{\prime}$ s as in Example 6.1.

2. Assume an annual premium of $P=700$ paid at the beginning of year; 10 -year term; policy issued to 60-year-old.

3. Death benefit at end of year of death (state 2$)=5000 ; S_{t+1}^{(02)}=S_{t+1}^{(12)}=5000$.

4. Disability benefit at end of year if disabled (state 1 ) at end of year is $B_{t+1}^{(1)}=750$.

5. Premiums paid at beginning of year if healthy.

6. Endowment of 1000 in healthy state 0 at $t=10$.

To compute probabilities, we will use Kolmogorov's equations. Please note that

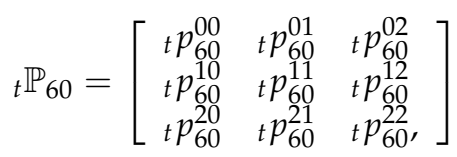

and 


$$
\begin{aligned}
\mathbb{Q}_{60+t} & =\left[\begin{array}{lll}
-\mu_{60+t}^{01}-\mu_{60+t}^{02} & \mu_{60+t}^{01} & \mu_{60+t}^{02} \\
\mu_{60+t}^{10} & -\mu_{60+t}^{10}-\mu_{60+t}^{12} & \mu_{60+t}^{12} \\
\mu_{60+t}^{20} & \mu_{60+t}^{20} & -\mu_{60+t}^{20}-\mu_{60+t}^{21}
\end{array}\right] \\
= & {\left[\begin{array}{ccc}
-0.05-0.025 t & 0.05 & 0.025 t \\
0.025 & -0.025-0.04 t & 0.04 t \\
0 & 0 & 0
\end{array}\right] . }
\end{aligned}
$$

Kolmogorov's equations are as follows:

$$
\frac{d}{d t}\left({ }_{t} \mathbb{P}_{60}\right)={ }_{t} \mathbb{P}_{60} \mathbb{Q}_{60+t} ; \quad{ }_{0} \mathbb{P}_{60}=I_{3},
$$

for $t=1,2,3, \ldots, 9,10$. This can be solved easily using MATLAB. Next, we compute the recursion for the policy value ${ }_{t} V^{(j)}$. That is,

$$
\begin{aligned}
{ }_{t} V^{(0)}= & \sum_{k=0}^{2} p_{60+t}^{0 k} \cdot v\left(B_{t+1}^{(k)}+S_{t+1}^{(0 k)}+V_{t+1}^{(k)}\right)-P_{t}^{(0)} \\
= & p_{60+t}^{00} \cdot v\left(B_{t+1}^{(0)}+S_{t+1}^{(00)}+V_{t+1}^{(0)}\right)+v p_{60+t}^{01}\left(B_{t+1}^{(1)}+S_{t+1}^{(01)}+V_{t+1}^{(1)}\right) \\
& \quad \quad v p_{60+t}^{02}\left(B_{t+1}^{(2)}+S_{t+1}^{(02)}+V_{t+1}^{(2)}\right)-700 \\
= & v p_{60+t}^{00} V_{t+1}^{(0)}+v p_{60+t}^{01} V_{t+1}^{(1)}+v p_{60+t}^{02} V_{t+1}^{(2)}+v p_{60+t}^{01}(750)+v p_{60+t}^{02}(5000)-700 \\
= & v p_{60+t}^{00} V_{t+1}^{(0)}+v p_{60+t}^{01} V_{t+1}^{(1)}+v p_{60+t}^{01}(750)+v p_{60+t}^{02}(5000)-700
\end{aligned}
$$

and

$$
\begin{aligned}
{ }_{t} V^{(1)} & =\sum_{k=0}^{2} p_{60+t}^{1 k} \cdot v\left(B_{t+1}^{(k)}+S_{t+1}^{(1 k)}+V_{t+1}^{(k)}\right)-P_{t}^{(1)} \\
& =v p_{60+t}^{10} V_{t+1}^{(0)}+v p_{60+t}^{(11)} V_{t+1}^{(1)}+\underbrace{v p_{60+t}^{12} V_{t+1}^{(2)}}_{=0}+v p_{60+t}^{10}(0)+v p_{60+t}^{11}(750)+v p_{60+t}^{12}(5000) .
\end{aligned}
$$

Since ${ }_{t} V^{(2)}=0$ for all $t \geq 0$ there is no need for a recursion. Thus, we have the following recursion:

$$
\left[\begin{array}{c}
{ }_{t} V^{(0)} \\
{ }_{t} V^{(1)} \\
{ }_{t} V^{(2)}
\end{array}\right]=v \mathbb{P}_{60+t}\left[\begin{array}{c}
{ }_{t+1} V^{(0)} \\
{ }_{t+1} V^{(1)} \\
{ }_{t+1} V^{(2)}
\end{array}\right]+\left[\begin{array}{l}
v p_{60+t}^{01}(750)+v p_{60+t}^{02}(5000)-700 \\
v p_{60+t}^{11}(750)+v p_{60+t}^{12}(5000) \\
0
\end{array}\right],
$$

with the following terminal conditions for policy values:

$$
\left[\begin{array}{l}
{ }_{10} V^{(0)} \\
{ }_{10} V^{(1)} \\
{ }_{10} V^{(2)}
\end{array}\right]=\left[\begin{array}{c}
1000 \\
0 \\
0
\end{array}\right]
$$

Next, we compute the recursion for $\overrightarrow{{ }^{2} \sigma_{t}}$.

Recall the following:

$$
\overrightarrow{{ }^{2} \sigma_{t}}=\left[{ }^{2} \sigma_{t}^{(0)},{ }^{2} \sigma_{t}^{(1)},{ }^{2} \sigma_{t}^{(2)}\right]
$$

and 


$$
\overrightarrow{2 \sigma_{t}^{d}}=\left[2 \sigma_{t}^{(0), d}, 2 \sigma_{t}^{(1), d}, 2 \sigma_{t}^{(2, d)}\right]
$$

From Corollary 1,

$$
{ }^{2} \sigma_{t}^{(0), d}=v^{2} \sum_{k \neq l} p_{60+t}^{0 k} p_{60+t}^{0 l}\left(W_{t+1}^{0 k}-W_{t+1}^{0 l}\right)^{2},
$$

where

$$
\begin{gathered}
W_{t+1}^{00}=V_{t+1}^{(0)}+B_{t+1}^{(0)}-S_{t+1}^{(00)}=V_{t+1}^{(0)}, \\
W_{t+1}^{01}=V_{t+1}^{(1)}+750-0=V_{t+1}^{(1)}+750,
\end{gathered}
$$

and

$$
W_{t+1}^{02}=V_{t+1}^{(2)}+B_{t+1}^{(2)}-S_{t+1}^{(02)}=-5000
$$

Thus,

$$
{ }^{2} \sigma_{t}^{(0), d}=v^{2}\left(p_{60+t}^{00} p_{60+t}^{01}\left(V_{t+1}^{(0)}-V_{t+1}^{(1)}-750\right)^{2}+p_{60+t}^{00} p_{60+t}^{02}\left(V_{t+1}^{(0)}+5000\right)^{2}+v^{2}\left(p_{60+t}^{01} p_{60+t}^{02}\left(V_{t+1}^{(1)}+5750\right)^{2}\right) .\right.
$$

We also have the following:

$$
{ }^{2} \sigma_{t}^{(1), d}=v^{2} \sum_{k \neq l} p_{60+t}^{1 k} p_{60+t}^{1 l}\left(W_{t+1}^{1 k}-W_{t+1}^{1 l}\right)^{2}
$$

where

$$
\begin{gathered}
W_{t+1}^{10}=V_{t+1}^{(0)}+B_{t+1}^{(0)}-S_{t+1}^{(10)}=V_{t+1}^{(0)}, \\
W_{t+1}^{11}=V_{t+1}^{(1)}+750-0=V_{t+1}^{(1)}+750,
\end{gathered}
$$

and

$$
W_{t+1}^{12}=V_{t+1}^{(2)}+B_{t+1}^{(2)}-S_{t+1}^{(12)}=-5000
$$

So,

${ }^{2} \sigma_{t}^{(1), d}=v^{2}\left(p_{60+t}^{10} p_{60+t}^{11}\left(V_{t+1}^{(0)}-V_{t+1}^{(1)}-750\right)^{2}+p_{60+t}^{10} p_{60+t}^{12}\left(V_{t+1}^{(0)}+5000\right)^{2}+v^{2}\left(p_{60+t}^{11} p_{60+t}^{12}\left(V_{t+1}^{(1)}+5750\right)^{2}\right)\right.$.

Additionally,

$$
{ }^{2} \sigma_{t}^{(2), d}=0
$$

The recursion for ${ }^{2} \sigma_{t}$ is given by

$$
\overrightarrow{{ }^{2} \sigma_{t}}=\left[\begin{array}{c}
{ }^{2} \sigma_{t}^{(0)} \\
{ }^{2} \sigma_{t}^{(1)} \\
{ }^{2} \sigma_{t}^{(2)}
\end{array}\right]=v^{2} \mathbb{P}_{60+t} \cdot{ }^{2} \sigma_{t+1}+{ }^{2} \overrightarrow{\sigma_{t+1}^{d}} .
$$

Terminal conditions: $\overrightarrow{{ }^{2}} \overrightarrow{\sigma_{10}}=\overrightarrow{0}$.

We end the example by stating the following:

1. $\mathbb{P}_{t}$ is obtained from solving the Kolmogorov equations in forward time with initial conditions. 
2. ${ }_{t} V$ is obtained from solving the annual recursion for ${ }_{t} V^{(j)}$ backwards in time with terminal conditions.

3. $\overrightarrow{{ }^{2} \sigma_{t}}$ is obtained from solving the annual recursion for $\overrightarrow{{ }^{2} \sigma_{t}}$ backwards in time with terminal conditions.

4. All the previous three steps can be easily accomplished using MATLAB or similar software.

\section{Conclusions}

In this paper, we have explicitly derived a Hattendorff differential equation that describes the continuous time evolution of the variance of the loss at time $t$ random variable given that it is in state $j$ denoted by ${ }^{2} \sigma_{t}^{(j)}$ for continuous cash flows, along with terminal conditions. In addition, we have also derived a Hattendorff recursion for the annual and $h$-yearly evolution of ${ }^{2} \sigma_{t}^{(j)}$ for annual cash and $h$-yearly cash flows respectively, along with terminal conditions. Both derivations were carried out in the general setting of a multistate Markov insurance model. In both cases, the equations are coupled with policy values which are in turn solutions of either the Thiele's differential equation in continuous time or a policy value recursion in discrete time.

We have also developed a matrix notation to easily adapt the differential equation and recursion for programming into MATLAB or similar software. We have shown the adaptation and closed form expressions for the differential equation and recursion for two examples. Differential equations and recursions are easily solved using MATLAB and hence, the setting lends itself into easily programmable calculations for ${ }^{2} \sigma_{t}^{(j)}$.

We conclude by stating that all four objectives stated in the beginning of this paper have been achieved. In addition, to our knowledge, such an explicit derivation of both the continuous and discrete time models governing the evolution of ${ }^{2} \sigma_{t}^{(j)}$ that is very conducive for computations on MATLAB has not been shown before. In a future paper, we will demonstrate the use of the development in this paper to create numerical routines that can be adapted for a variety of insurance problems.

Author Contributions: Both authors contributed equally to this paper on everything. All authors have read and agreed to the published version of the manuscript.

Funding: This research received no external funding.

Institutional Review Board Statement: Not applicable.

Informed Consent Statement: Not applicable.

Data Availability Statement: Not applicable.

Conflicts of Interest: The authors declare no conflict of interest.

\section{Abbreviations}

The following abbreviations are used in this manuscript:

MDPI Multidisciplinary Digital Publishing Institute

DOAJ Directory of open access journals

TLA Three letter acronym

LD Linear dichroism

\section{Appendix A. Assumptions and Notations}

We introduce the following notation for states $i$ and $j$ and ages $x \geq 0$ as in (Dickson et al. 2020, chp. 8):

\section{Notation A1.}

1. ${ }_{t} p_{x}^{i j}=\operatorname{Pr}[Y(x+t)=j \mid Y(x)=i]$. This is probability that state of the process at time $t$ is $j$ given that the state at time 0 is $i$. 
2. ${ }_{t} p_{x}^{\overline{i i}}=\operatorname{Pr}[Y(x+s)=i$ for all $s \in[0, t] \mid Y(x=i)]$. This is the probability that the state resides continuously in state ifrom time 0 to time $t$.

3. Force of transition: $\mu_{x}^{i j}=\lim _{h \rightarrow 0^{+}} \frac{h p_{x}^{i j}}{h}$ for $i \neq j$.

4. $\quad L_{t}^{(j)}=$ Future loss random variable for a policy which is in state $j$ at time $t$

5. $V_{t}^{(j)}=E\left[L_{t}^{(j)}\right]$. Expected present value of the future loss random variable at time $t$ given that the state at time $t$ is $j$.

6. $\sigma_{t}^{2(j)}=\operatorname{Var}\left[L_{t}^{(j)}\right]$. Variance of future loss random variable at time $t$ given that the state at time $t$ is $j$.

For simplicity of notation, we will simply write $Y(t)$ instead of $Y(x+t)$, with the assumption that the policyholder is of age $x$ at $t=0$. We also make the following assumptions as in (Dickson et al. 2020, chp. 8):

\section{Assumption A1.}

1. (Markovian assumption) For any states $i, j$ and times $t$ and $t+s$, the conditional probability $\operatorname{Pr}[Y(t+s)=j \mid Y(t)=i]$ is well defined and is independent of the knowledge of the process before time $t$.

2. The probability of two or more transitions in a time interval $h$ is o $(h)$.

3. For all states $i$ and $j$ and all ages $x \geq 0$, we assume that $t_{x}^{i j}$ is a differentiable function of $t$.

4. For simplicity, we assume that there are no expenses although expenses can be easily incorporated into the formulation.

5. For simplicity, we assume a constant interest rate $i$ although a variable interest can be easily incorporated into the formulation.

We also make the following assumptions and notation on the payments for $h$-yearly cash flows:

\section{Notation A2.}

1. We assume that for the discrete time payments, the payments depend at most on the state of the process $Y(t)$ at the start and end of the period between cash flows.

2. $h P_{t}^{(j)}$ - amount of premium payable at the start of the interval $(t, t+h)$ given that $Y(t)=j$. $P_{t}^{(j)}$ is a premium rate which is differentiable with respect to $t$.

3. $h B_{t+h}^{(k)}$ - benefit payable at the end of the interval $(t, t+h)$ given that $Y(t+h)=k . B_{t}^{(k)}$ is a benefit rate which is differentiable with respect to $t$. Additionally, for consistency we have

$$
\lim _{h \rightarrow 0} B_{t+h}^{(k)}=-P_{t}^{(k)} .
$$

We allow for the mathematical possibility that $B_{t+h}^{(k)}$ and $P_{t}^{(k)}$ both exist in the time interval $(t, t+h)$ as benefit rate at the end given $Y(t+h)=k$ and premium rate at the beginning given $Y(t)=k$ respectively, with the understanding that one or both can be zero for a given state $k$.

4. $S_{t+h}^{(j k)}$-lump sum benefit payable at the end of the interval $(t, t+h)$ if $Y(t+j)=k$, given that $Y(t)=j$. Here we allow $S_{t+h}^{(j k)}$ to be differentiable with respect to $t$ and allow $j=k$ as a mathematical possibility as well, with the assumption that for small $h, S_{t+h}^{(j j)}=h B_{t+h^{\prime}}^{(j)}$ and hence

$$
\lim _{h \rightarrow 0} S_{t+h}^{(j j)}=0 ; \quad \lim _{h \rightarrow 0} \frac{S_{t+h}^{(j j)}}{h}=B_{t}^{(j)} .
$$

We include terms of the form $S_{t}^{(j j)}$ for symmetry in the derivation, with the understanding that $S_{t}^{(j j)}=0$ since $S_{t}^{(j k)}$ is in principle, a benefit paid upon transition from state $j$ to state $k$ with $j \neq k$, and hence is equal to zero when $j=k$. 
5. We define $W_{t+h}^{(j k)}=V_{t+h}^{(k)}+h B_{t+h}^{(k)}-S_{t+h}^{(j k)}$ and $W_{t}^{(j k)}=\lim _{h \rightarrow 0} W_{t+h}^{(j k)}=V_{t}^{(k)}-S_{t}^{(j k)}$.

We introduce some matrix and vector notation that will be useful in Section 4 .

\section{Notation A3.}

1. Represent the variance vector as

$$
\overrightarrow{{ }^{2} \sigma_{t}}=\operatorname{Var}\left(L_{t}\right)=\left[2 \sigma_{t}^{(0)},{ }^{2} \sigma_{t}^{(1)}, \ldots,{ }^{2} \sigma_{t}^{(m)}\right]^{T} .
$$

2. Let the $(i, j)$ th element of the matrix ${ }_{h} \mathbb{P}$ be given by

$$
\left({ }_{h} \mathbb{P}_{x+t}\right)_{i j}={ }_{h} p_{x+t}^{i j} .
$$

3. In this light, we have

$$
{ }^{2} \sigma_{t+h}^{(j), d}=\operatorname{Var}\left(L_{t+h}^{(j), d}\right)=v^{2 h} \sum_{k \neq l} h p_{x+t h}^{j k} p_{x+t}^{j l}\left(W_{t+h}^{(j k)}-W_{t+h}^{(j l)}\right)^{2},
$$

where

$$
L_{t+h}^{(j), d}=v^{h} W_{t+h}^{(j k)} \quad \text { with probability }{ }_{h} p_{x+t}^{j k} \text { and } \sum_{k=0}^{m}{ }_{h} p_{x+t}^{j k}=1 .
$$

4. Define the vector of variance of losses as

$$
\overrightarrow{{ }^{2} \sigma_{t}^{d}}=\operatorname{Var}\left(L_{t}^{d}\right)=\left[{ }^{2} \sigma_{t}^{(0), d},{ }^{2} \sigma_{t}^{(1), d}, \ldots,{ }^{2} \sigma_{t}^{(m), d}\right]^{T}
$$

5. Define the j-th element of the vector $\mu_{x+t}^{\vec{d}}$ as

$$
\left\{\mu_{x+t}^{\vec{d}}\right\}_{j}=\sum_{l \neq j} \mu_{x+t}^{j l}\left(W_{t}^{(j j)}-W_{t}^{(j l)}\right)^{2} .
$$

6. Define the $(i, j)$-th element of the matrix $\mathbb{M}_{x+t}$ as

$$
\left(\mathbb{M}_{x+t}\right)_{i j}=\left\{\begin{array}{lr}
2 \delta+\sum_{k \neq l} \mu_{x+t}^{j k} & \text { if } i=j \\
-\mu_{x+t}^{i j} & \text { if } i \neq j
\end{array} .\right.
$$

7. $\mathbb{M}$ is in terms of known matrices. That is, define

$$
v_{x+t}^{i}=\sum_{l=0}^{m} \mu_{x+t}^{i l},
$$

then define the $(m+1) \times(m+1)$ transition intensity matrix

$$
\left\{\mathbb{Q}_{x+t}\right\}_{i j}=\left\{\begin{array}{ll}
-v_{x+t}^{i} & \text { if } i=j \\
\mu_{x+t}^{i j} & \text { if } i \neq j
\end{array} .\right.
$$

So,

$$
\mathbb{M}_{x+t}=2 \delta I_{m+1}-\mathbb{Q}_{x+t},
$$

where $I_{m+1}$ represents the $(m+1) \times(m+1)$ identity matrix.

8. Define the matrix discount factor as

$$
{ }_{l}^{2} \mathbb{E}_{x+t}=v^{2 l}{ }_{l} \mathbb{P}_{x+t} .
$$


The matrix discount factor can be factored. Recall that

$$
{ }_{m}^{2} \mathbb{E}_{x+t}=v^{2 m} \mathbb{P}_{x+t}
$$

and

$$
{ }_{n}^{2} \mathbb{E}_{x+t}=v^{2 n}{ }_{n} \mathbb{P}_{x+t}
$$

Therefore,

$$
\begin{aligned}
{ }_{m}^{2} \mathbb{E}_{x+t} \cdot{ }_{n}^{2} \mathbb{E}_{x+t+m} & =v^{2 m}{ }_{m} \mathbb{P}_{x+t} \cdot v^{2 h}{ }_{n} \mathbb{P}_{x+t+m} \\
& =v^{2(m+n)}{ }_{m} \mathbb{P}_{x+t} \cdot{ }_{n} \mathbb{P}_{x+t+m} \\
& =v^{2(m+n)}{ }_{m+n} \mathbb{P}_{x+t} \\
& ={ }_{m+n}^{2} \mathbb{E}_{x+t} .
\end{aligned}
$$

Since $v$ and $p$ satisfy $|v|<1$ and $\left.\right|_{n} p_{x+t}^{i j} \mid<1$, we have the following:

$$
\lim _{n \rightarrow \infty} \frac{2}{n} \mathbb{E}_{x+t}=0 .
$$

\section{References}

Asmussen, Søren, and Mogens Steffensen. 2020. Risk and Insurance. Berlin/Heidelberg: Springer.

Bladt, Mogens, Søren Asmussen, and Mogens Steffensen. 2020. Matrix representations of life insurance payments. European Actuarial Journal 10: 29-67. [CrossRef]

Bowers, Newton L., Hans U. Gerber, James C. Hickman, Donald A. Jones, and Cecil J. Nesbitt. 1986. Actuarial Mathematics. Itasca: The Society of Actuaries.

Dickson, David C. M., Mary R. Hardy, and Howard R. Waters. 2020. Actuarial Mathematics for Life Contingent Risks. Cambridge: Cambridge University Press.

Gerber, Hans U. 1979. An Introduction to Mathematical Risk Theory. Philadelphia: Huebner Foundation, University of Pennsylvania.

Gerber, Hans U. 1986. Lebellsversicherungsmathematik. Berlin/Heidelberg: Springer.

Hattendorff, Karl. 1868. Das risiko bei der lebenversicherung. Masius Rundschau der Versi 18: 169-83.

Norberg, Ragnar. 1992. Hattendorff's theorem and thiele's differential equation generalized. Scandinavian Actuarial Journal 1: 2-14. [CrossRef]

Norberg, Ragnar. 1995. Differential equations for moments of present values in life insurance. Insurance: Mathematics and Economics 17: 171-80. [CrossRef]

Papatriandafylou, Alex, and Howard R. Waters. 1984. Martingales in life insurance. Scandinavian Actuarial Journal 1984: 210-30. [CrossRef]

Ramlau-Hansen, Henrik. 1988. Hattendorff's theorem: A markov chain and counting process approach. Scandinavian Actuarial Journal 1988: 143-56. [CrossRef]

Wolthuis, Henk. 1987. Hattendorff's theorem for a continuous-time markov model. Scandinavian Actuarial Journal 1987: 157-75. [CrossRef] 